\title{
Global Software development: An Approach to Design and Evaluate the Risk factors for Global Practitioners
}

\author{
Chamundeswari Arumugam \\ Department of Computer Science and Engineering \\ SSN College of Engineering \\ Tamil Nadu, India \\ chamundeswaria@ssn.edu.in
}

\author{
Baskaran Kaliamourthy \\ Managed Services, Engineer Manager \\ Ericsson Inc, Plano, \\ Texas, USA \\ baskaran@outlook.in
}

\begin{abstract}
In today's competitive environment organizations are increasingly moving towards global software development to utilize every possible benefits across geography. Practitioners from different part of the world are undertaking such assignments for multiple benefits like global exposure, new domain/arena to explore, better compensation, etc. In spite of the above benefits these global practitioners face multiple challenges due to cultural difference, communication, geographical dispersion.
\end{abstract}

This paper emphasizes the risk factors on global practitioners in an IT organization. Dependencies of the global practitioners are explored to identify the risk factors using four stages of dynamics model [30]. A Socio Technical Systems (STS) responsibility model is designed for global practitioners to exhibit the relationship between global practitioners within an organization structure and represent the risk of the global practitioners using a set of graphical notations. In addition this paper also proposes a MultiAgent Simulation Model (MASM) to evaluate the risk of global practitioners on gaining trust from project stakeholders. This work highlights the sequential and concurrent activities based on schedule and time for evaluating the risk.

Keywords - global practitioners; socio technical system; multiagent simulation model; global system development

\section{INTRODUCTION}

The rapid advancement in the Information Technology (IT) has widely spread the use of the Global Software Development (GSD) across the world. GSD is popular among the IT organization and a large number of IT employees are taking up global assignment, due to its benefits it offers, irrespective of the duration. Reduced development costs, leveraging time-zone effectiveness, cross site modularization of development work, access to large skilled labor pool, innovation and shared best practice, and close proximity to market and customer are some of the key benefits of GSD [6]. Many IT employees around the global are migrating from one region to another irrespective of the culture, language, security, food, etc. for GSD projects, and they are termed as global practitioners in this paper.

DOI reference number : 10.18293/SEKE2016-077
Many organizations have set up their branches in many cost economical different countries to outsource their projects. GSD differs from Distributed Software Development (DSD), as the development happens across national geographic boundaries. Development of projects across national geographic boundaries sets the cause of all challenges in GSD for global practitioners.

Global practitioners can be categorized as independent workers and employees of globally distributed companies. The challenges are different across these two categories. This paper focuses on the challenges of global practitioners in globally distributed companies. Global practitioners play a vital role in every phase of software development life cycle. This paper attempts to take a look at the risk associated with global practitioner in GSD projects, to gain trust of all stakeholders through conceptual model and Socio Technical System (STS) towards designing responsibility model to identify the risk for global practitioners and thereafter the possibility to apply MultiAgent Simulation Model (MASM) to evaluate them. The MASM can be used to evaluate the probability and impact of the risk by the global practitioners, to gain trust from stakeholders.

The organization of this paper is as follows. Section II provides an overview of related work in GSD risk and STS. Section III explains the research methodology in detail. Finally, Section IV concludes the paper and discusses limitations as well as future work.

\section{RESEARCH METHODOLOGY}

Global practitioners are selected by the organization based on the experience level, skill set, work division, domain knowledge, competency level, domain experience, time zone difference, cultural difference, process maturity diversity, critical functionality, commitment, etc. The selected global practitioners, has risk to gain trust from stakeholders in a GSD team.

The four stage dynamics model specified in Pressman [23] are considered as information resources to identify the risk factors. Forming, storming, norming, and performing are the four stages used as information resources to identify the risk 
factors. During forming stage, an effective work culture should be set, ignoring conflicts and assigning commitment among the members. Setting up, work culture is a risk and it need to done to ensure commitment.

In storming stage, a well growth oriented foster relationship should be set, with close mind members in a project. Also the members should organize task and add value to the team. Setting up a foster relationship is risk and it is advisable, as each member can add value to his team. In norming stage, transparent process should be encouraged, to permit team members to take decision. Encouraging transparent process is risk but it enhances knowledge sharing.

\section{TABLE I. IDENTIFICATION OF RISK FACTORS}

\begin{tabular}{|l|l|l|}
\hline $\begin{array}{l}\text { Information } \\
\text { resource }\end{array}$ & Risk factor & Dependent factors \\
\hline forming & work culture & $\begin{array}{l}\text { conflicts }^{-} \\
\text {commitment }^{+}\end{array}$ \\
\hline storming & $\begin{array}{l}\text { foster } \\
\text { relationship }\end{array}$ & $\begin{array}{l}\text { close minds }^{-} \\
\text {organize task }^{+} \\
\text {member value }^{+}\end{array}$ \\
\hline norming & $\begin{array}{l}\text { transparent } \\
\text { process }\end{array}$ & $\begin{array}{l}\text { knowledge sharing }^{+} \\
\text {team feedback }\end{array}$ \\
\hline performing & Visibility & $\begin{array}{l}\text { shared goals } \\
\text { optimal solution }\end{array}$ \\
\hline
\end{tabular}

In performing stage, more visibility needs to be created, as they work on shared goals to produce optimal solution. Creating a visibility is a risk, but it promotes to produce optimal solutions. The identified risk factors are represented in Table 1.

Conceptualization of a problem is the most important activity in the development of a system dynamics model [21]. Scholl in 1995 [22] implied that four mapping tools, causal loop diagrams, stock and flow diagrams, sector diagrams, and policy structure diagrams, are frequently combined in system dynamics projects. In this work, the identified risk factors are conceptualized using causal loop diagram. Vensim tool [24] is used to represent the conceptualization model using causal loop diagram. Causal loop diagram is represented in Figure 1. Using the identified risk factors, the STS responsibility model is derived and it is discussed below.

The concept of a "socio-technical system" (as part of "organizational development" research) was created in the 1960s by E. Trist and F. Emery (both of the Tavistock Institute for Social Research in London). This term "socio-technical system" basically considers the interaction of people in a social system or organization, with tools and technique, in a technical system. Baxter [2] specified six key characteristics of sociotechnical systems. Cherns [5] stated four key principles of socio-technical systems.

Responsibility modeling [13] is a graphical modeling and analysis technique designed to help people record and analyze responsibilities within organizations, to explore the structure and dependability of socio-technical systems. Russell lock et al. [10] specified the key components of a responsibility model are responsibilities, agents, and shared information. In this paper, responsibility modeling technique is used to represent the design of the identified risk factors. The key components identified for the responsibility model are information resource, risk factor, and multiagent. Here the multiagent is used to analyze and access the interactions among the various risk factors that happen between four information resources.

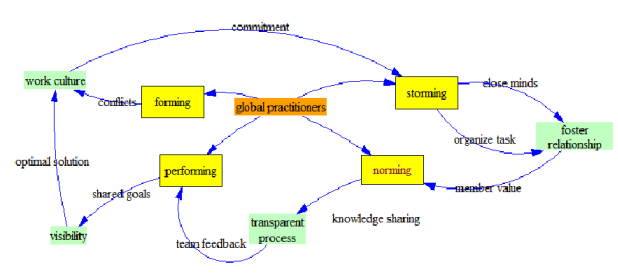

Figure 1. Conceptual model using causal loop diagram

The key component uses five symbols to represent relationship. The five symbols actually used for representation are curved rectangle, parallel line, greater and lesser sign, filled in circle, filled in diamond. Curved rectangle represents risk factor, parallel lines denote information resource, and greater and lesser sign denote multiagent. The other two symbols, filled in circle and hollow diamond are used to represent the relationship between risk factor, information resource, and multiagent. Figure. 2 represent the STS responsibility model for three key components with their corresponding relationship.

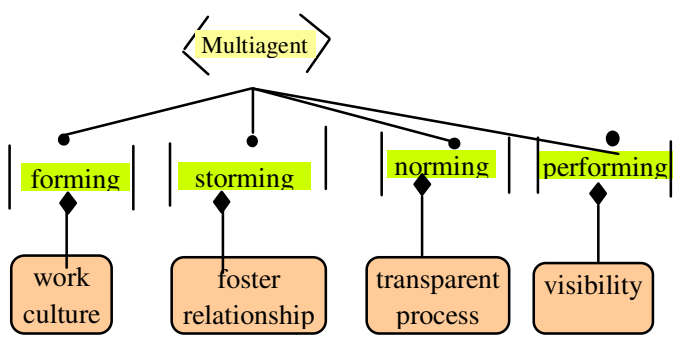

Figure 1. Responsibility model

\section{A. Risk Assessment Model (RAM)}

Multi-Agent Simulation Model (MASM) can provide a way for stakeholders to experiment with the different configurations [7]. Agent based simulation was already applied in software development projects for simulation purpose to forecast the performance of individual team members in a software development team [1]. The goal of MASM in this proposed work is to evaluate the information resources dynamic changes for risk assessment.

MASM was practically applied in software industry in certain environment where the complete development may procure cost. Considering this factor, an attempt has been done in this paper to apply MABS for risk assessment. Here, MASM can be applied to assess the risk for the global practitioners to gain trust from stakeholders, considering the four information resources to simulate the dynamic data. A 
complex relationship prevails between the risk factors, and dependent factors, onto which multiagents can be applied. Figure 3. represents the agents flow direction to collect the data from the identified risk factors for risk evaluation.

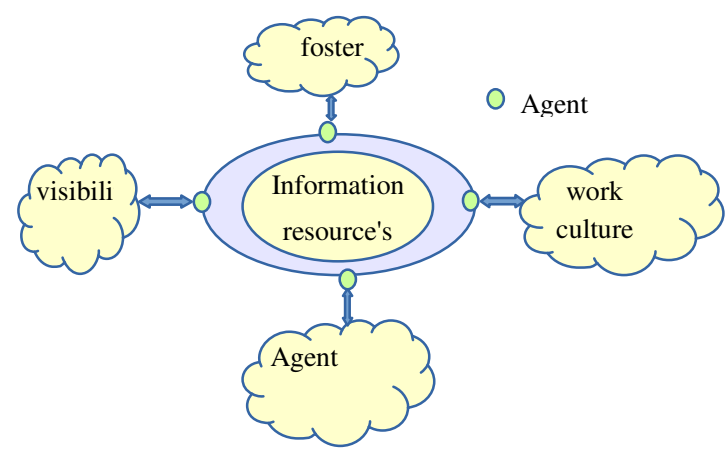

Figure 2. Flow of agent to collect the data from information resource

A single agent can perform one or more activities associated with information resources for risk assessment. An activity correspond a set of tasks to assess the risk. The simplest task represents a single evaluation in RAM. Each activity is attached with constraints. The constraints can be sequential order or time based event. The nesting of the events in sequential order and time event can be complex and this is referred as concurrent activities. Each agent is independent, and it has the responsibility to start an activity and also has privilege to interact with one or more agents. In multiagent concept, for interaction, the agents mutually agree and proceed. An agent has the right to accept or reject the interaction.

Figure. 4 show the proposed approach of using MASM for assessing the risk, a Risk Assessment Model (RAM). An agent associated with an information resource has many sequential and concurrent activities. An agent through execution control interacts either with sequential or concurrent activities of model, while data analysis collects and record the data for the various interaction between agents, for risk assessment.

\section{LITERATURE SURVEY}

Nils Brede Moe et al. [3] indicated that trust is a fundamental factor in determining the success or failure of GSD projects. Some of the identified key factors to be poor socialization and socio-cultural fit increased monitoring, inconsistency and disparities in work practices, reduction of and unpredictability in communication, lack of face-to-face meetings, language skills, conflict handling, and cognitivebased trust. The effect of lacking trust has implication for software managers, and practitioners involved in GSD. Christof Ebert et al. [8] identify and mitigate the GSE risk for development projects and product evolution.

Responsibility modeling $[10,12]$ is a graphical modeling technique designed to allow end users to model and explore many of the high level risks within their socio-technical systems without recourse to expensive risk management specialists. Indira Nurdiani et al. [11] conducted a systematic review on GSD literature and gathered challenges associated with GSD projects as well as their mitigation strategies. The authors developed two static checklists for risk identification and mitigation. However, the author pointed out, they cannot be used as decision making tool to select the most appropriate mitigation for particular risk. Ansgar Lamersdorf et al. [9] assessed risks for task allocation alternatives based on project and site-specific characteristics and analyzed it with respect to possible project risks stemming from the work distribution.

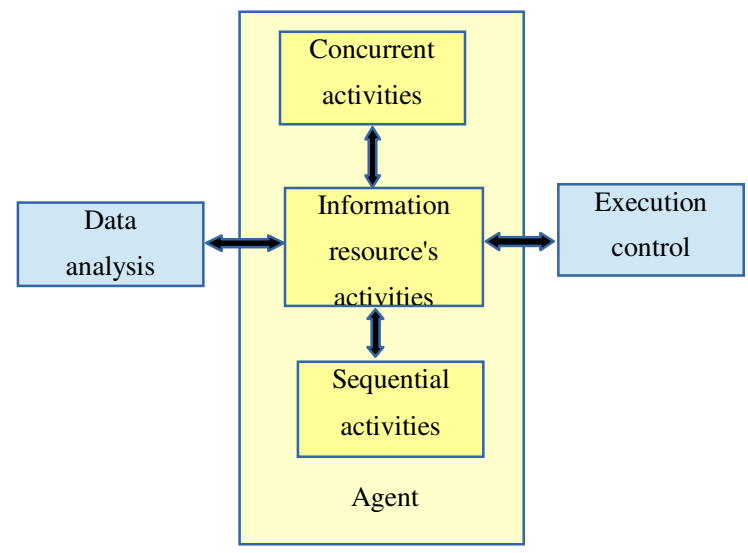

Figure 3. Risk assessment model using MABS

Verner et al. [15] has investigated GSD to discover the risk and mitigation advice for the organizations involved with GSD. The authors extracted 85 risks and 77 risk mitigation advice items and categorized them under four major headings: outsourcing rationale, software development, human resources, and project management. Muhammad Usman et al. [14] identified communication is a very important risk factor in GSD. Davy de Medeiros Baia [7] aims to create an integrated multi-agent-based simulation to support software project management.

Mohammed Alshammri [17] presents a research plan to develop combined agent-based and system dynamics models to simulate software development process focusing on the behaviour of team members in a software project environment. Kellner et al. [18] has clearly stated why, what, how to simulate. Wickenberg et al. [19] investigated the applicability of multiagent based simulation to simulate software development processes. David et al. [20] provided a set of general guidelines when to use simulation in software development process.

From the literature work, it is understood, identification and mitigation of risk based on GSD is important in GSD projects. However, in the present scenario, the global practitioners are the important contributor in GSD project. Thus, this paper proposes an approach to design and evaluate the risk factors, for a global practitioners in a GSD team to gain the trust of the stakeholders.

\section{CONCLUSION}

This paper attempt to take a look at the risk associated with global practitioner in the GSD projects to gain trust from stakeholders. This paper proposes an approach to identify and 
assess the risk factors, for global practitioners in GSD projects using four stage dynamics model [30]. A conceptual model that combines the dynamics for global practitioners in GSD projects, using the four stages, forming, norming, storming and performing, is attempted to identify the risk. Causal loop diagram mapping tool captures the dynamics and it is represented using the Vensim tool. Design using a socio technical responsibility modeling technique is represented to exhibit the relationship between global practitioners within an organization using a set of notations. Also, Risk Assessment Model (RAM) using MultiAgent Simulation Model (MASM) design is proposed to assess the risk factors. Assessing these risk factors is a measure for the global practitioners in the GSD projects.

\section{ACKNOWLEDGMENT}

We thank Dr. S. Sivakumar, Professor, SSN College of Engineering, Tamil Nadu, India for his continuous support and value added comments in drafting this paper.

\section{REFERENCES}

[1] R. Agarwal, D. Umphress. "A Flexible Model for Simulation of Software Development Process," $48^{\text {th }}$ Annual Southeast Regional Conference, 4 pages. 2010, New York, NY: ACM.

[2] G. Baxter, I. Sommerville, Socio-technical systems: From design methods to systems engineering. Submitted to The journal of humancomputer studies, 2008.

[3] N.B. Moe, D. Smite, "Understanding a Lack of Trust in Global Software Teams: A Multiple-case Study," Software Process Improvement and Practice, vol 13, pp 217-231, 2008.

[4] I.Bider, H.Otto,

[5] A.B. Cherns, The principles of socio technical design, Human Relations, vol. 29 (1976), pp. 783-792.

[6] E. Ó Conchúir, P. J. Ågerfalk, H. H. Olsson, and B. Fitzgerald, "Global software development: where are the benefits?", Communications of the ACM, vol. 52, pp. 127-131, 2009.

[7] D. D. M. Baia, "An Integrated Multi-Agent-Based Simulation Approach to Support Software Project Management," 2015 IEEE/ACM 37th IEEE International Conference on Software Engineering, Doctoral Symposium, 2015.

[8] C. Ebert, B. K. Murthy, N.N. Jha, "Managing Risks in Global Software Engineering: Principles and Practices," IEEE International Conference on Global Software Engineering, 2008, pp.131-140.
[9] A. Lamersdorf, J. Münch, A. F. V. Torre, C.R. Sánchez, "A Risk-driven Model for Work Allocation in Global Software Development Projects," 2011 Sixth IEEE International Conference on Global Software Engineering, pp. 15-24.

[10] R.Lock, I.Sommerville , "Modelling and Analysis of Socio-Technical System of Systems", 15th IEEE International Conference on Engineering of Complex Computer Systems, 2010, pp. 224-232.

[11] I. Nurdiani , R. Jabangwe, D.Smite, D.Damian, "Risk Identification and Risk Mitigation Instruments for Global Software Development: Systematic Review and Survey Results," Sixth IEEE International Conference on Global Software Engineering Workshops, pp. 36-41, 2011

[12] R. Lock, I. Sommerville, Socio Technical Systems Engineering Handbook.

[13] D. Šmite and J. Borzovs, "Managing Uncertainty in Globally Distributed Software Development Projects," University of Latvia, Computer Science and Information Technologies, vol. 733, pp. 9-23, 2008.

[14] M. Usman, F. Azam, N. Hashmi, Analysing and reducing risk factor in 3-C's model communication phase used in global software development, International Conference on Information Science and Applications (ICISA), pp. 1-4 (2014)

[15] J. M. Verner, O.P. Brereton, B.A. Kitchenham, M. Turner, M. Niazi, Risks and risk mitigation in global software development: A tertiary study Information and Software Technology, 56 (2014), pp. 54-78.

[16] A. Zeid and R. El-Bahey, "Establishing a global software development course: A cultural perspective" , Frontiers in Education Conference, pp.1695 -1701, 2013.

[17] M. Alshammri, Simulation Modelling of Human Aspects in Software Project Environment, ASWEC ' 15 Vol. II, September 28 - October 01, 2015, Adelaide, SA, Australia.

[18] M. I. Kellner, Raymond J. Madachy, and David M. Raffo, "Software Process Modeling and Simulation: Why, What, How," Journal of Systems and Software, Vol. 46, No. 2/3 (15 April 1999).

[19] T. Wickenberg, and P. Davidsson. 2003. "On Multi Agent Based Simulation of Software Development Processes." In Multi-Agent-Based Simulation II, Edited by J. Simão Sichman, F. Bousquet, and P. Davidsson, vol. 2581, 72-77, Berlin: Springer Berlin-Heidelberg.

[20] D. Joslin, and W. Poole, Agent-Based Simulation For Software Project Planning, Proceedings of the 2005 Winter Simulation Conference.

[21] R. Eberlein, V. Diker, R. Langer, J. Rowe (Eds.), Proceedings of the 21 st International Conference of the System Dynamics Society. July 2024, 2003 New York City, NY (2003).

[22] Scholl, G. J. (1995). "Benchmarking the system dynamics community: research results." System Dynamics Review 11(2): 139 to 155.

[23] Pressman, R. Software Engineering: A Practitioner's Approach. McGraw-Hill, 2005)

[24] www.vensim.com 